\title{
Tratamiento de drenaje ácido de mina con el uso de zeolita natural a escala experimental
}

\section{Treatment of acid mine drainage with the use of natural zeolite on an experimental scale}

\section{Moreno Balseca, Alex Rodrigo}

Ingeniero Ambiental, Universidad Central del Ecuador armoreno@uce.edu.ec

\section{Palacios Cabrera, Teresa}

Docente Facultad de Ingeniería en Geología, Minas, Petróleos y Ambiental de la Universidad Central del Ecuador tapalacios@uce.edu.ec

\section{Resumen}

El presente proyecto de investigación se realizó en el Instituto Nacional de Investigación Geológico Minero Metalúrgico (INIGEMM) con un total de 40 litros de agua del Drenaje Ácido de Mina (AMD, por sus siglas en inglés) recolectados en la Quebrada “El Panteón”, Parroquia Torata, Cantón Santa Rosa, Provincia de El Oro.

El AMD se trató mediante un sistema de tratamiento que consistió en una serie de dos columnas de lixiviación conectadas por flujo a través de una bomba peristáltica, las cuales contenían materiales reactivos tipo Sustrato Alcalino Disperso (DAS, por sus siglas en inglés), y la adición de zeolita natural como un medio filtrante. En el sistema de tratamiento se realizaron 12 mediciones de conductividad y $\mathrm{pH}$, mientras que, para el análisis de metales (Al, $\mathrm{Fe}, \mathrm{Cu}, \mathrm{Pb}, \mathrm{Zn}, \mathrm{Mn}$ y Cd) y el anión SO42- se realizaron cuatro mediciones.

El valor de $\mathrm{pH}$ promedio de AMD tratado fue 8.03 y la conductividad promedio obtenida fue de $3254 \mu \mathrm{S} / \mathrm{cm}$. La eficiencia de remoción promedio tratada fue: $\mathrm{Al}$ (97.81\%); $\mathrm{Fe}$ (99.79\%); $\mathrm{Cu}(91.42 \%) ; \mathrm{Pb}$ (83.33\%); $\mathrm{Zn}(99.84 \%) ; \mathrm{Mn}$ (96.14\%); Cd (88.54\%) y SO42- (51.47\%). El sistema presenta una alta remoción de metales conforme a los resultados obtenidos, por lo que la descarga cumple con la normativa ambiental nacional, exceptuando el anión $\mathrm{SO}_{4}{ }^{2-}$.

Palabras clave: drenaje ácido de mina; zeolita natural; escala experimental; metales pesados; sustrato alcalino disperso

\section{Abstract}

The present research project was carried out at the Instituto Nacional de Investigación Geológico Minero Metalúrgico (INIGEMM) with a total of 40 liters of water of Acid Mine Drainage (AMD) taken from the Quebrada "El Panteón", Parroquia Torata, Cantón Santa Rosa, Provincia de El Oro.

The AMD was treated through a treatment system which consisted of a series of two leach columns connected by flow through a peristaltic pump, which contained a mixture of reactive materials type Dispersed Alkaline Substrate (DAS), and the addition of natural zeolite as a filter medium.

In the treatment system, 12 measurements of conductivity and $p H$ were taken, while four measurements were taken for the analysis of metals ( $\mathrm{Al}, \mathrm{Fe}, \mathrm{Cu}, \mathrm{Pb}, \mathrm{Zn}, \mathrm{Mn}$, and $\mathrm{Cd})$ and SO42- anion.

A final average of $p H$ of treated $A M D$ was 8.03 and a final average conductivity was 3,254mS/cm. The final aver-age removal efficiency were $\mathrm{Al}$ (97.81\%); $\mathrm{Fe}$ (99.79\%); $\mathrm{Cu}$ (91.42\%); $\mathrm{Pb}$ (83.33\%); $\mathrm{Zn}$ (99.84\%); $\mathrm{Mn}$ (96.14\%); Cd (88.54\%) and SO42- (51.47\%). The system has a high metal removal according to the results obtained, so the discharge complies with the national environmental regulations, except for the anion SO42-.

Keywords: acid mine drainage; natural zeolite; experimental scale; heavy metals; dispersed alkaline substrate 


\section{Introducción}

El Drenaje Ácido de Mina (AMD, por sus siglas en inglés) es el resultado de la oxidación de sulfuros metálicos, en presencia de oxígeno atmosférico y agua como lo indica la Red Internacional de Prevención de Ácidos (INAP, por sus siglas en inglés) [1]; además el AMD da origen a lixiviados ácidos altamente contaminantes con acidez y altas concentraciones de $\mathrm{Fe}, \mathrm{Cu}, \mathrm{Zn}, \mathrm{Pb}, \mathrm{Cd}, \mathrm{Mn}, \mathrm{As}$, etc. [2].

El AMD es uno de los principales problemas causados por las actividades mineras y tiene el potencial de contaminar aguas superficiales y subterráneas [3]. La descarga incontrolada de aguas de mina con concentraciones elevadas de contaminantes en el medio ambiente puede afectar la vida acuática, el suelo, y los sedimentos [4]. La importancia de la propuesta de un sistema de tratamiento pasivo para el AMD, a escala experimental, radica en llenar el vacío investigativo en cuanto a dar alternativas de tratamiento eficientes y menos costosas, para de esta manera impedir que el AMD generado por procesos de minería de metales, genere impactos negativos hacia el ambiente y población.

Los sistemas de tratamiento pasivo se han convertido en una de las formas más sostenibles y factibles de remediar el AMD. Sin embargo, los tratamientos convencionales muestran obstrucción temprana de la porosidad y/o revestimiento de los materiales reactivos cuando se tratan altas concentraciones de acidez y metales contenidos en el AMD [5]; Ref.

[6] denomina a estos procesos como pasivación (pérdida de reactividad debido al recubrimiento) y/o obstrucción (pérdida de permeabilidad) por la formación de precipitados. El denominado Sustrato Alcalino Disperso (DAS, por sus siglas en inglés), es un sistema de tratamiento pasivo probado para el tratamiento del AMD que se compone de un reactivo alcalino de grano fino (arena de calcita) mezclado con un material inerte de tamaño grueso con alta superficie específica (viruta de madera), sistema que muestra un alto éxito en cuanto a rendimiento químico e hidráulico probado en columnas de laboratorio [6].

Un estudio experimental para la eliminación de metales divalentes contenidos en el AMD como el Zn, $\mathrm{Cu}, \mathrm{Pb}$ y $\mathrm{Mn}$ en condiciones de $\mathrm{pH}$ entre 3 y 5,5 con columnas rellenas de magnesia caustica ( $\mathrm{MgO}$ ) y arena de cuarzo (tamaño de grano de $2-4 \mathrm{~mm}$ ) se llevó a cabo dando resultados favorables de remo- ción de dichos metales [7]. También se realizó un estudio experimental mezclando virutas de madera con diferentes tamaños de grano de $\mathrm{MgO}$ para tratar aguas con concentraciones altas de $\mathrm{Zn}$ y $\mathrm{Mn}$, dando resultados favorables de eliminación de estos metales [8].

La zeolita natural es un mineral aluminosilicato hidratado cristalino que por sus propiedades de porosidad, adsorción e intercambio iónico ha sido ampliamente utilizado para la remoción de metales. Investigaciones para la aplicabilidad de zeolita natural para la remoción de metales pesados en solución contenidos en el AMD han sido estudiados por varios autores, obteniendo resultados favorables para la remoción de metales como el $\mathrm{Fe}, \mathrm{Cu}, \mathrm{Zn}$, $\mathrm{Mn}, \mathrm{Pb}, \mathrm{Cd}$, etc. [9], [10], [11]; por lo que su uso es una alternativa como un sustituto de los adsorbentes más caros como carbón activado u otros métodos de adsorción de metales.

El proyecto se realizó en las instalaciones del Instituto Nacional de Investigación Geológico Minero Metalúrgico (INIGEMM) con muestras de AMD tomadas de la Quebrada "El Panteón", en la Parroquia Torata, Cantón Santa Rosa de la Provincia de El Oro. Las muestras de AMD se llevaron al sistema de tratamiento pasivo propuesto que consistía en una serie de dos columnas de lixiviación conectadas por flujo a través de una bomba peristáltica, estas columnas contenían una mezcla de materiales reactivos tipo DAS y la adición de zeolita natural como un medio filtrante.

El objetivo de esta investigación fue realizar análisis físico químicos, como el potencial de hidrógeno $(\mathrm{pH})$ y conductividad eléctrica (CE), mediante el muestreo de varios puntos en el sistema de tratamiento pasivo propuesto para el análisis del comportamiento de estos parámetros físico químicos y las condiciones que hacen posible la remoción de la concentración de los metales y aniones en estudio.

\section{Metodología experimental}

\section{Muestreo del drenaje ácido de mina}

\section{De inición de puntos de muestreo}

Se realizó la visita de campo al sitio de estudio en la Quebrada "El Panteón", Parroquia Torata, Cantón Santa Rosa, Provincia de El Oro (Fig. 1, p. 3), en donde se tomaron dos muestras simples en recipientes de $20 \mathrm{~L}$, denominadas como Recipiente $1 \mathrm{y}$ Recipiente 2 (Tabla 1). 
Tabla 1. Ubicación de puntos de muestreo

\begin{tabular}{|c|c|c|c|}
\hline \multirow{2}{*}{ Código muestra } & \multicolumn{3}{|c|}{ Coordenadas (UTM WGS84) } \\
\cline { 2 - 4 } & $\mathbf{x}$ & $\mathbf{y}$ & $\mathbf{z}$ \\
\cline { 2 - 4 } & $\mathbf{m}$ & $\mathbf{~ m}$ & $\mathbf{m}$ \\
\hline Recipiente 1 & 627453 & 9604980 & 612 \\
\hline Recipiente 2 & 626553 & 9602962 & 298 \\
\hline \multicolumn{4}{|c}{ Elaborado por: Alex Moreno }
\end{tabular}

El muestreo se realizó en dos campañas correspondientes a época seca del sector, el primer muestreo correspondiente al Recipiente 1 tomado el 03/12/2015 se ubicó a la salida de la bocamina del frente de explotación "Ecuaba" de la concesión minera "El Guayabo", la cual drenaba AMD a la Quebrada "El Panteón", mientras que el segundo muestreo correspondiente al Recipiente 2 tomado el 07/11/2016 se ubicó en la Quebrada "El Panteón", aguas abajo respecto al primer muestreo, antes de la confluencia de la quebrada con el Río Santa Rosa; se pudo observar que de acuerdo al Catastro Minero Nacional proporcionado por el INIGEMM, el área del sector de estudio se encontraba bajo concesiones mineras de minerales metálicos (Oro-Plata).

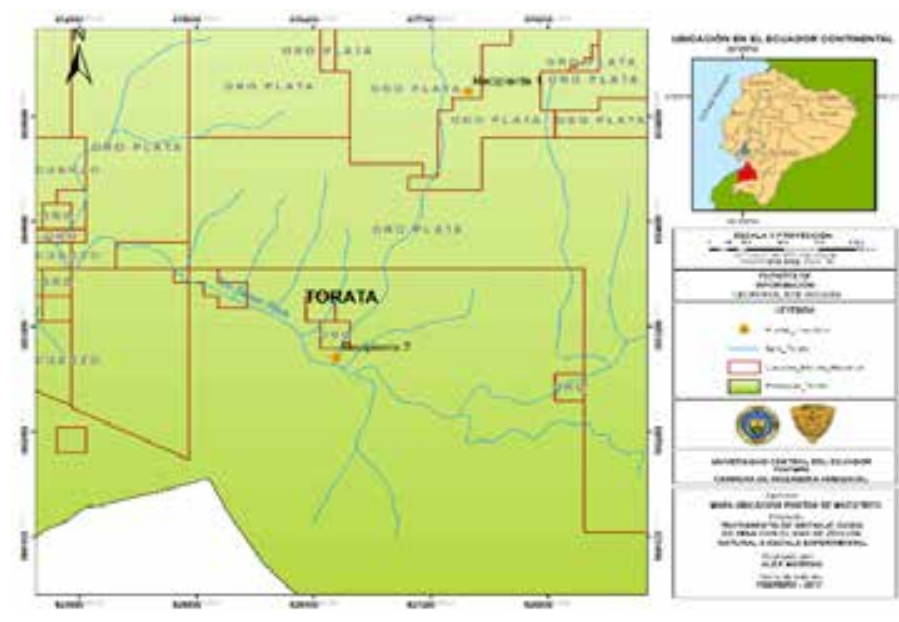

Figura 1. Mapa de ubicación de puntos de muestreo

\section{Protocolo de muestreo}

El protocolo para la toma de muestras de AMD fue el siguiente:

1. En campo se ubicó el punto de muestreo previamente seleccionado; una vez identificado, se levantó información del lugar donde se desarrolló el muestreo.

2. I dentificado el punto de muestreo, se procedió a seleccionar el sector representativo del sitio de muestreo correspondiente.

3. Procedimiento de toma de muestras:
Se ubicó en el punto seleccionado.

Se tomó el recipiente limpio (recipientes de $20 \mathrm{~L}$ ) y se llenó con el AMD que posteriormente se analizará.

El recipiente fue lavado tres veces con el AMD.

Se sumergió el recipiente y se llenó evitando hojas, raíces, o cualquier interferencia que pueda interferir con los resultados.

Durante el llenado, la boca del recipiente se orientó en sentido contrario a la corriente.

Una vez llenado el recipiente, se levantó rápidamente y se tapó de inmediato, evitando cámaras de aire dentro del mismo.

Los recipientes fueron etiquetados como "Recipiente 1" y "Recipiente 2" utilizando marcador permanente, éstos se cubrieron con cinta adhesiva transparente, evitando el borrado de la información.

4. Las muestras tomadas fueron enviadas a los laboratorios del INIGEMM, las cuales fueron almacenadas a temperatura ambiente (180C) hasta su uso en el sistema de tratamiento pasivo.

\section{Análisis de parámetros isicoquímicos del drenaje ácido de mina}

Se tomó una muestra simple del recipiente 1 y 2 en un vaso de precipitación de vidrio de $500 \mathrm{~mL}$. Los parámetros medidos fueron $\mathrm{pH}$ y $\mathrm{CE}$, mediante un multiparámetro, fabricante HANNA, modelo HI 9829, éste fue controlado y calibrado usando tres soluciones estándar de pH (4-7-10) y una solución estándar de conductividad (1000 $\mu \mathrm{S} / \mathrm{cm})$.

\section{Análisis de concentración de metales del drenaje ácido de mina}

Se tomaron dos muestras simples en envases plásticos de $500 \mathrm{~mL}$ para el análisis de metales y aniones correspondientemente, éstas fueron ingresadas para su análisis al laboratorio del INIGEMM.

Para la medición de metales se utilizó el espectrofotómetro de emisión óptica con plasma acoplado inductivamente (ICP-OES), fabricante PERKIN ELMER, modelo Óptima 8300 y el análisis de aniones se realizó mediante el cromatógrafo iónico compacto para aniones, fabricante METROHM, modelo Compact ICPRO 881. Los metales considerados fueron $\mathrm{Al}, \mathrm{Cd}, \mathrm{Cu}, \mathrm{Fe}$, $\mathrm{Mn}, \mathrm{Pb}$ y $\mathrm{Zn}$; mientras que el anión analizado fue el SO42-. Los límites de detección (LD) en mg/L fueron: $\mathrm{Al}(0,19)$; Cd, Cu, Fe, Mn, Pb, Zn y SO42 $(0,01)$. 


\section{Diseño e instalación del sistema de tratamiento pasivo}

El diseño e instalación del sistema de tratamiento pasivo propuesto constó de un tanque que contenía el AMD inicial, el cual se conectó a una serie de dos columnas de lixiviación, denominadas DAS-Ca y DAS-Mg de material polimetilmetacrilato de aproximadamente $45 \mathrm{~cm}$ de altura y $10 \mathrm{~cm}$ de diámetro interno conectada por flujo mediante una bomba peristáltica; éstas finalmente se conectaron a un tanque final que contenía el AMD tratado. La zeolita natural fue lavada con agua destilada y secada en estufa a 110 oC durante 24 horas, además se realizó un análisis granulométrico a través de tamizado en seco mediante una tamizadora vibratoria marca Retsch, modelo AS 200.

El análisis mineralógico de la zeolita natural se determinó mediante una pistola de fluorescencia de rayos $X(F R X)$, marca NITON, modelo XL3t 500, con autocalibración y en modo "Granal Normal", LD de 1 ppm para todos los elementos. Las mediciones se realizaron seis veces con una duración de 90 segundos cada una a fin de obtener el promedio de la medición de cada elemento. La zeolita natural fue colocada a manera de lecho en la columna DAS-Mg.

\section{Muestreo de agua y análisis de parámetros fisicoquímicos del sistema de tratamiento pasivo}

Se realizaron un total de 12 muestreos (tres muestreos por semana aproximadamente) durante un mes en vasos de precipitación de vidrio de $50 \mathrm{~mL}$ a lo largo del sistema de tratamiento pasivo instalado, para el análisis fisicoquímico in situ se establecieron tres puntos específicos denominados como: AMD inicial, AMD-Ca-Mg (situado en el sobrenadante DAS-Mg) y AMD tratado.

Los parámetros medidos fueron $\mathrm{pH}$ y $\mathrm{CE}$, mediante un multiparámetro HANNA-HI 9829, éste fue contro-lado y calibrado mediante las recomendaciones del fabricante y usando tres soluciones estándar de $\mathrm{pH}$ (4-7-10) y una solución estándar de conductividad $(1000 \mu \mathrm{S} / \mathrm{cm})$.

\section{Análisis de concentración de metales del sistema de tra- tamiento pasivo}

En el sistema de tratamiento pasivo en los puntos AMD inicial y AMD tratado, se realizaron cuatro muestreos (uno por semana) con toma de cuatro muestras cada uno (2 de AMD inicial y 2 de AMD tratado), recolectados en envases plásticos de 500 $\mathrm{mL}$ para el posterior análisis de metales y aniones.

Para la medición de metales de la primera muestra se utilizó ICP-OES, mientras que la medición de me- tales de las siguientes muestras se realizó en los laboratorios de la Facultad de Ingeniería en Geología, Minas, Petróleos y Ambiental (LABFIGEMPA) utilizando el espectrofotómetro de absorción atómica (EAA) Perkin Elmer modelo PinAAcle 900T con LD en mg/L: Al (1,1); Cd (0,03); Cu $(0,08)$; Fe $(0,1)$; Mn $(0,05) ; \mathrm{Pb}(0,19)$ y $\mathrm{Zn}(0,02)$; mientras que el anión analizado fue el SO42- mediante el cromatógrafo iónico compacto METROHM, Compact ICPRPO 881.

Los parámetros obtenidos en la presente investigación son analizados tomando en cuenta la Reforma al Anexo 1 del Libro VI del Texto Unificado de Legislación Secundaria del Ministerio del Ambiente (TULSMA), Acuerdo Ministerial 097, Tabla 9: LÍMITES DE DESCARGA A UN CUERPO DE AGUA DULCE (referido en adelante como TULSMA), mediante la cual se estableció una comparación entre los valores obtenidos del muestreo con los límites permisibles indicados en la normativa ambiental nacional [12].

Finalmente para comparar la eficiencia de remoción de los metales y aniones del sistema de tratamiento pasivo se utilizaron los resultados obtenidos del muestreo del AMD inicial y AMD tratado mediante la Ec. (1).

\section{$P R=\left(A M D\right.$ inicial-AMD tratado)/(AMD inicial) ${ }^{\star} 100 \quad E c .(1)$}

Donde:

PR: Porcentaje de remoción (\%)

AMD inicial: Concentración inicial del AMD (mg/L)

AMD tratado: Concentración final del AMD tratado $(\mathrm{mg} / \mathrm{L})$

\section{Resultados y discusión}

\section{Muestreo del drenaje ácido de mina}

Se trabajó con un total de $40 \mathrm{~L}$ de AMD correspondientes a dos recipientes de $20 \mathrm{~L}$ cada uno, las cuales se utilizaron para el ingreso en el sistema de tratamiento pasivo. En los sitios de muestreo se observaron precipitados amarillo-rojizo-marrón, además el sitio contenía aguas de coloración amarillo-marrón seguramente causada por una abundancia de partículas suspendidas de hidróxidos de hierro.

\section{Análisis de parámetros isicoquímicos del drenaje ácido de mina}

Los resultados del análisis fisicoquímico de las muestras de los recipientes 1 y 2 se indican en la tabla 2. 
Tabla 2. Resultados de parámetros fisicoquímicos del AMD inicial

\begin{tabular}{|c|c|c|c|}
\hline Código muestra & $\mathbf{t}\left({ }^{\circ} \mathbf{C}\right)$ & $\mathbf{p H}$ & $\mathbf{C E}(\boldsymbol{\mu S} / \mathbf{c m})$ \\
\hline Recipiente 1 & 21,50 & 2,61 & 2617 \\
\hline Recipiente 2 & 21,60 & 2,71 & 5861 \\
\hline
\end{tabular}

\section{Potencial de hidrógeno (pH)}

El $\mathrm{pH}$ de las muestras analizadas tanto en el recipiente 1 y 2 varía entre 2,61 y 2,71 (Tabla 2), $\mathrm{pH}<6$, obteniéndose un AMD de tipo ácido por lo que se considera que la acidez generada pudo haberse dado por oxidación de minerales, particularmente sulfuros asociado a minas metálicas, carbón y piritas [13], (citado en [14], p. 2); además la observación de precipitados amarillo-marrón en el sitio de estudio es típica para el proceso de AMD como lo indica Lottermoser [4].

Una vez que el AMD entra en contacto con la atmósfera, éste se oxigena y mediante un proceso aerobio catalizado por bacterias de tipo Thiobacillus ferrooxidans, en un ambiente de $\mathrm{pH}$ entre 2 y 3,5; transforman el hierro ferroso a hierro férrico, dando una coloración ocre-rojo intenso al agua [14].

En comparación con los valores de pH establecidos en el TULSMA las muestras presentan valores de $\mathrm{pH}$ por debajo de los límites mínimo (pH 6) y máximo (pH 9) permisibles.

\section{Conductividad eléctrica (CE)}

La conductividad medida varía entre 5861 a 2617 $\mu \mathrm{S} / \mathrm{cm}$ en los recipientes 1 y 2 . Los valores de conductividad medidos corresponden posiblemente al alto contenido de iones y metales en solución, puesto que el AMD da origen a lixiviados ácidos contaminantes con altas concentraciones de sulfatos y metales como Fe, Cu, Zn, Pb, Cd, Mn, As, etc. [2].

\section{Análisis de metales y aniones del drenaje ácido de mina}

Los resultados del análisis de metales y aniones en las muestras del recipiente 1 se indican en la Tabla 3 (p. 5).

\section{Tabla 3. Resultados de análisis de metales y aniones en AMD inicial del recipiente 1}

\begin{tabular}{|c|c|c|c|}
\hline $\begin{array}{c}\text { Código } \\
\text { muestra }\end{array}$ & $\begin{array}{c}\text { Paráme- } \\
\text { tros }\end{array}$ & $\begin{array}{c}\text { Resultados } \\
\text { análisis (mg/L) }\end{array}$ & $\begin{array}{c}\text { Criterio cali- } \\
\text { dad (TULS- } \\
\text { MA) (mg/L) }\end{array}$ \\
\hline
\end{tabular}

\begin{tabular}{|c|c|c|c|}
\hline & $\mathrm{Al}$ & 24,6 & 5,0 \\
\cline { 2 - 4 } & $\mathrm{Cd}$ & 0,14 & 0,02 \\
\cline { 2 - 4 } AMD- & $\mathrm{Cu}$ & 4,2 & 1,0 \\
\cline { 2 - 4 }$-\mathrm{T}-001$ & $\mathrm{Fe}$ & 200,6 & 10,0 \\
\cline { 2 - 4 } & $\mathrm{Mn}$ & 12,0 & 2,0 \\
\cline { 2 - 4 } & $\mathrm{Pb}$ & 0,2 & 0,2 \\
\cline { 2 - 4 } & $\mathrm{Zn}$ & 14,5 & 5,0 \\
\hline \multicolumn{3}{|c|}{ Fuente: MAE, 2015 } \\
\hline
\end{tabular}

Los resultados indican altas concentraciones de metales, metaloides y aniones ( $\mathrm{Fe}, \mathrm{Cu}, \mathrm{Zn}, \mathrm{Pb}$, $\mathrm{Cd}, \mathrm{Mn}, \mathrm{SO}_{4}^{2-}$ etc.), esto indica que existió la oxidación de sulfuros metálicos, especialmente los de hierro (piritaFeS ${ }_{2}$ ) en presencia de oxígeno atmosférico y agua [1], [2], [4]. En cuanto al recipiente 2 , éste fue ingresado al sistema de tratamiento pasivo una vez el sistema tuvo ausencia de AMD en el punto AMD inicial.

Se observa que los elementos analizados no cum-plen con los criterios de calidad de la reforma al TULSMA, a excepción del Pb.

\section{Diseño e instalación del sistema de tratamiento pasivo}

Se utilizaron columnas de flujo descendente abiertas a la atmósfera (Fig. 2) a fin de maximizar la oxidación del Fe2+ y minimizar la movilidad del mismo [6].

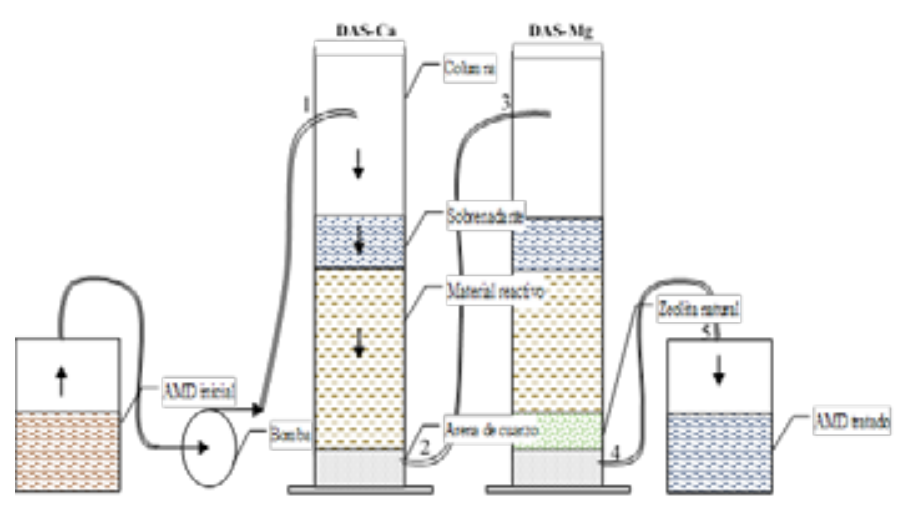

Figura 2. Diseño del sistema de tratamiento pasivo.

Se realizaron 5 orificios en el sistema de tratamiento, los cuales se describen a continuación: El orificio 1 correspondía a la entrada a la columna DAS-Ca (a $25 \mathrm{~cm}$ de altura desde la base); el orificio 2 a la salida de la columna DAS-Ca (a $2 \mathrm{~cm}$ de altura desde la base); el orificio 3 a la entrada a la columna DAS-Mg (a $25 \mathrm{~cm}$ de altura desde la base); el orificio 4 a la salida de la columna DAS-Mg (a $2 \mathrm{~cm}$ de altura desde la base) y el punto 5 a la entrada al tanque final (centro de entrada al tanque). En todos los puntos se realizaron cortes en las mangueras instaladas a manera de drenes (excepto el punto 5), para que el AMD fluya constantemente. Los componentes del sistema de tratamiento pasivo propuesto se detallan 
a continuación:

AMD inicial: Corresponde al tanque inicial que contiene el AMD a tratar mediante el sistema de tratamiento pasivo, a este tanque se ingresó el AMD del recipiente 1 y a medida de AMD faltante, se ingresó AMD del recipiente 2.

Bomba: Se utilizó una bomba peristáltica de laboratorio marca LONGERPUMP, modelo BT100-1L, la cual provee el AMD desde el tanque AMD inicial hacia la primera columna DAS-Ca con un caudal constante de aproximadamente $1 \mathrm{~L} /$ día.

DAS-Ca: Dentro de la primera columna de lixiviación denominada DAS-Ca, se añadió arena de cuarzo a manera de lecho; además se añadió una mezcla de viruta de madera (longitud aproximada $4 \mathrm{~mm}$ ) con calcita (CaCO3), el cual comprende el denominado material reactivo. La mezcla fue homogeneizada durante 15 minutos y se dejó en reposo 2 días. El líquido que se mantenía de manera superficial luego del ingreso del AMD se denominó como sobrenadante. La función de esta columna es disminuir la concentración de Fe y Al que necesitan condiciones de $\mathrm{pH}$ de 6 a 7 [7]. Ref. [6], en un estudio experimental en columnas de laboratorio indica que la mayor parte del ion $\mathrm{Fe} 3+$ disuelto se precipitó cerca de la superficie del material reactivo disminuyendo así su concentración, de la misma manera ocurrió con el aluminio, el cual precipitó a mayor profundidad $(3 \mathrm{~cm})$ con respecto al hierro. Los pesos y alturas correspondientes a la columna DAS-Ca se muestran en la Tabla 4.

\section{Tabla 4. Composición de columna DAS-Ca}

\begin{tabular}{|c|c|c|}
\hline Material & Peso (g) & Altura (cm) \\
\hline Arena de cuarzo & 300 & 2,9 \\
\hline Viruta de madera & 90,7 & \multirow{2}{*}{14} \\
\hline $\mathrm{CaCO}_{3}$ & 22,68 & \\
\hline
\end{tabular}

DAS-Mg: La columna denominada DAS-Mg comprendió un lecho de arena de cuarzo, y una mezcla de viruta de madera (longitud aproximada $2 \mathrm{~mm}$ ) con óxido de magnesio ( $\mathrm{MgO}$ ), denominado como material reactivo. La mezcla fue homogeneizada durante 15 minutos y se dejó en reposo 2 días. La columna DAS-Mg funciona de tal manera que disminuye la concentración de metales divalentes $(\mathrm{Zn}, \mathrm{Mn}, \mathrm{Cu}$, $\mathrm{Pb}$, y $\mathrm{Cd}$ ) debido a que la disolución del $\mathrm{MgO}$ eleva el $\mathrm{pH}$ a valores comprendidos entre 8 y 10 [7]. La zeolita natural tipo clinoptilolita (proporcionada por la Empresa Vital del Ecuador) fue añadida a la columna DAS-Mg con un tamaño de grano aproximado de 0,5 a $1 \mathrm{~mm}$ (Tabla 5, p.6). Este mineral posee la capacidad de intercambio de iones presentes en los metales disueltos en el AMD [4]. Además puede neutralizar soluciones ácidas; esto se logra mediante el intercambio de $\mathrm{H}+$ de la solución con los cationes intercambiables en la estructura de zeolita [15].

Tabla 5. Resultado de análisis granulométrico de la clinoptilolita

\begin{tabular}{|c|c|c|}
\hline $\begin{array}{c}\text { Peso inicial (g) } \\
\text { Abertura malla (\# } \\
\text { malla) }\end{array}$ & $\begin{array}{c}\text { Peso de la } \\
\text { fracción (g) }\end{array}$ & $\%$ \\
\hline $1 \mathrm{~mm}(\# 18)$ & 83,73 & 56,04 \\
\hline $500 \mu \mathrm{m} \mathrm{( \# 35)}$ & 63,48 & 42,55 \\
\hline $355 \mu \mathrm{m} \mathrm{( \# 45)}$ & 0,33 & 0,22 \\
\hline $250 \mu \mathrm{m}(\# 60)$ & 0,10 & 0,07 \\
\hline $180 \mu \mathrm{m}(\# 80)$ & 0,03 & 0,02 \\
\hline $125 \mu \mathrm{m}(\# 120)$ & 0,00 & 0,00 \\
\hline $90 \mu \mathrm{m}(\# 170)$ & 0,00 & 0,00 \\
\hline $63 \mu \mathrm{m}(\# 230)$ & 0,07 & 0,05 \\
\hline $45 \mu \mathrm{m}(\# 325)$ & 0,51 & 0,34 \\
\hline Base & 1,17 & 0,78 \\
\hline TOTAL & 149,42 & 100 \\
\hline
\end{tabular}

Elaborado por: Alex Moreno

El análisis mineralógico de la clinoptilolita (Tabla 6) muestra que su composición mayoritaria es de $\mathrm{Ca}$, Fe y $\mathrm{K}$ con un 40,88\%; 30,59\% y 18,78\% respectivamente.

\section{Tabla 6. Resultado de análisis mineralógico de la clinoptilolita}

\begin{tabular}{|c|c|c|c|}
\hline No. & Elemento & ppm & $\%$ \\
\hline 1 & $\mathrm{Ca}$ & 12701,46 & 40,88 \\
\hline 2 & $\mathrm{Fe}$ & 9505,57 & 30,59 \\
\hline 3 & $\mathrm{~K}$ & 5834,87 & 18,78 \\
\hline 4 & $\mathrm{Ti}$ & 1322,67 & 4,26 \\
\hline 5 & $\mathrm{~S}$ & 348,42 & 1,12 \\
\hline 6 & $\mathrm{Sr}$ & 341,80 & 1,10 \\
\hline 7 & $\mathrm{Ba}$ & 243,92 & 0,79 \\
\hline 8 & $\mathrm{Mn}$ & 219,83 & 0,71 \\
\hline 9 & $\mathrm{Zr}$ & 104,19 & 0,34 \\
\hline 10 & $\mathrm{Co}$ & 91,76 & 0,30 \\
\hline 11 & $\mathrm{Te}$ & 73,91 & 0,24 \\
\hline 12 & $\mathrm{Cs}$ & 47,88 & 0,15 \\
\hline 13 & $\mathrm{~V}$ & 41,56 & 0,13 \\
\hline 14 & $\mathrm{Sn}$ & 32,82 & 0,11 \\
\hline 15 & $\mathrm{Zn}$ & 30,57 & 0,10 \\
\hline 16 & $\mathrm{Sc}$ & 27,36 & 0,09 \\
\hline 17 & $\mathrm{Sb}$ & 24,43 & 0,08 \\
\hline 18 & $\mathrm{Cr}$ & 19,00 & 0,06 \\
\hline 19 & $\mathrm{Rb}$ & 11,54 & 0,04 \\
\hline 20 & $\mathrm{~Pb}$ & 10,72 & 0,03 \\
\hline 21 & $\mathrm{Pd}$ & 10,55 & 0,03 \\
\hline & & & \\
\hline
\end{tabular}




\begin{tabular}{|c|c|c|c|}
\hline 22 & $\mathrm{Cd}$ & 8,99 & 0,03 \\
\hline 23 & $\mathrm{As}$ & 6,37 & 0,02 \\
\hline 24 & $\mathrm{U}$ & 6,18 & 0,02 \\
\hline 25 & $\mathrm{Ag}$ & 5,74 & 0,02 \\
\hline \multicolumn{3}{|c}{ Elaborado por: Alex Moreno } \\
\hline
\end{tabular}

Los pesos y alturas correspondientes a la columna DAS-Mg, se muestran en la Tabla 7.

\section{Tabla 7. Composición de columna DAS-Mg}

\begin{tabular}{|c|c|c|}
\hline Material & Peso (g) & Altura (cm) \\
\hline Arena de cuarzo & 300 & 2,9 \\
\hline Viruta de madera & 71,3 & \multirow{2}{*}{11} \\
\hline MgO & 17,8 & \\
\hline Zeolita natural & 296,9 & 3 \\
\hline
\end{tabular}

Elaborado por: Alex Moreno

AMD tratado: Comprende el tanque final que contiene el AMD tratado por el sistema de tratamiento pasivo.

\section{Análisis de parámetros isicoquímicos del sistema de tra-tamiento pasivo}

Los resultados del análisis fisicoquímico de las muestras se indican en la Tabla 8.

Tabla 8. Resultados de parámetros isicoquímicos del sistema de tratamiento pasivo

\begin{tabular}{|c|c|c|c|c|}
\hline $\begin{array}{c}\text { No. Medi- } \\
\text { ción in situ }\end{array}$ & $\begin{array}{c}\text { Punto mues- } \\
\text { treo }\end{array}$ & $\mathbf{t}\left({ }^{\circ} \mathbf{C}\right)$ & $\mathbf{p H}$ & $\begin{array}{c}\mathbf{C E} \\
\boldsymbol{\mu S} / \mathrm{cm}\end{array}$ \\
\hline \multirow{4}{*}{1} & AMD inicial & 20,80 & 2,58 & 2870 \\
\cline { 2 - 5 } & AMD-Ca-Mg & 21,23 & 9,64 & 2154 \\
\cline { 2 - 5 } & AMD tratado & 21,10 & 8,66 & 1275 \\
\hline \multirow{4}{*}{2} & AMD inicial & 20,80 & 2,63 & 2811 \\
\cline { 2 - 5 } & AMD-Ca-Mg & 20,34 & 8,25 & 1129 \\
\cline { 2 - 5 } & AMD tratado & 20,90 & 8,54 & 2458 \\
\hline \multirow{4}{*}{3} & AMD inicial & 21,10 & 2,66 & 2800 \\
\cline { 2 - 5 } & AMD-Ca-Mg & 20,78 & 7,98 & 1642 \\
\cline { 2 - 5 } & AMD tratado & 21,08 & 8,26 & 2504 \\
\hline \multirow{4}{*}{4} & AMD inicial & 21,60 & 2,71 & 5861 \\
\cline { 2 - 5 } & AMD-Ca-Mg & 20,75 & 7,29 & 4078 \\
\cline { 2 - 5 } & AMD tratado & 20,81 & 7,87 & 3646 \\
\hline \multirow{4}{*}{5} & AMD inicial & 21,00 & 2,76 & 5120 \\
\cline { 2 - 5 } & AMD-Ca-Mg & 20,82 & 8,04 & 2571 \\
\cline { 2 - 5 } & AMD tratado & 20,90 & 8,15 & 2490 \\
\hline
\end{tabular}

\begin{tabular}{|c|c|c|c|c|}
\hline \multirow{4}{*}{6} & AMD inicial & 22,12 & 2,83 & 5063 \\
\cline { 2 - 5 } & AMD-Ca-Mg & 22,47 & 7,81 & 1781 \\
\cline { 2 - 5 } & AMD tratado & 21,42 & 8,09 & 2852 \\
\hline \multirow{4}{*}{7} & AMD inicial & 20,20 & 2,83 & 4930 \\
\cline { 2 - 5 } & AMD-Ca-Mg & 21,00 & 7,21 & 3800 \\
\cline { 2 - 5 } & AMD tratado & 21,00 & 7,84 & 2940 \\
\hline \multirow{4}{*}{8} & AMD inicial & 20,30 & 2,81 & 4980 \\
\cline { 2 - 5 } & AMD-Ca-Mg & 21,60 & 7,19 & 4078 \\
\cline { 2 - 5 } & AMD tratado & 20,70 & 7,59 & 3447 \\
\hline \multirow{4}{*}{10} & AMD inicial & 19,80 & 2,76 & 5750 \\
\cline { 2 - 5 } & AMD-Ca-Mg & 19,50 & 7,02 & 4910 \\
\cline { 2 - 5 } & AMD tratado & 20,10 & 7,74 & 4005 \\
\hline \multirow{4}{*}{11} & AMD inicial & 19,40 & 2,76 & 5755 \\
\cline { 2 - 5 } & AMD-Ca-Mg & 19,50 & 6,83 & 5385 \\
\cline { 2 - 5 } & AMD tratado & 19,70 & 7,86 & 4300 \\
\hline & AMD inicial & 21,00 & 2,76 & 5836 \\
\cline { 2 - 5 } & AMD-Ca-Mg & 21,10 & 7,01 & 5148 \\
\cline { 2 - 5 } & AMD tratado & 21,32 & 7,90 & 4421 \\
\hline \multirow{4}{*}{12} & AMD inicial & 20,41 & 2,75 & 5980 \\
\cline { 2 - 5 } & AMD-Ca-Mg & 19,88 & 7,06 & 4950 \\
\cline { 2 - 5 } & AMD tratado & 20,72 & 7,80 & 4715 \\
\hline
\end{tabular}

Elaborado por: Alex Moreno

\section{Potencial de hidrógeno (pH)}

AMD inicial: El valor de $\mathrm{pH}$ del AMD inicial varía entre 2,58 a 2,83; tomando en cuenta el TULSMA, estos valores se encuentran fuera de rango, tanto para el valor límite mínimo ( $\mathrm{pH}$ 6) como para el valor límite máximo ( $\mathrm{pH}$ 9) permisibles.

AMD-Ca-Mg: Se observa que en la primera medición in situ existe un aumento considerable de $\mathrm{pH}$ desde el AMD inicial al AMD-Ca-Mg, esto se debe a que la calcita de la columna DAS-Ca es consumida, elevando el pH desde 2,58 a 9,64 (Tabla 8, p. 6). Estos potenciales precipitarían a metales trivalentes ( $\mathrm{Fe}, \mathrm{Al}, \mathrm{Cr}$ ) que necesitan condiciones de $\mathrm{pH}$ de 6 a 7 y a metales divalentes ( $\mathrm{Zn}, \mathrm{Mn}, \mathrm{Cu}, \mathrm{Pb}$, $\mathrm{Ni}$, Co y $\mathrm{Cd}$ ) en condiciones de $\mathrm{pH}$ mayores a 8,5; los metales divalentes como el cinc precipitan en forma de Hidrocincita ( $\mathrm{Zn} 5(\mathrm{CO} 3) 2(\mathrm{OH}) 6)$, plomo en forma de hidróxido, cobre como hidroxisulfato y el $\mathrm{Mn} 2+$ se oxida y precipita como óxido de manganeso (Mn2O3) en forma de Manganita $(\mathrm{YMnOOH})$ [7], [8]. De manera similar, en las mediciones posteriores existe un aumento considerable de los valores de $\mathrm{pH}$ desde el AMD inicial al AMD-Ca-Mg, estableciéndose éste último en un rango promedio de 2,74 a 7,61 respectivamente.

AMD tratado: El AMD tratado tiene valores de $\mathrm{pH}$ entre 7,59 a 8,66 que son ligeramente superiores al AMD-Ca-Mg debido al proceso de desgasificación del CO2 [6], estos valores son significativamente al- 
tos comparados con el AMD inicial debido a la presencia de Brucita $(\mathrm{Mg}(\mathrm{OH}) 2)$ en la segunda columna denominada DAS-Mg, producto de la hidratación del reactivo $\mathrm{MgO}$ y por ende una alta concentración de $\mathrm{Mg}$, es importante también destacar que el tamaño pequeño y la gran superficie específica de las partículas de $\mathrm{MgO}$ (longitud 0,15 mm) son un factor importante en el rendimiento de la columna DAS-Mg en cuanto a remoción de metales como el Zn y Mn [8], [16]. Entonces, respecto al parámetro potencial de hidrógeno $(\mathrm{pH})$ medido para las muestras de AMD luego del tratamiento descrito, se logró obtener valores dentro del rango establecido en el TULSMA.

\section{Conductividad eléctrica (CE)}

AMD inicial: Existe variación en las mediciones de la conductividad, presentando un valor máximo en el AMD inicial de $5980 \mu \mathrm{S} / \mathrm{cm}$ y un mínimo de 2800 $\mu S / \mathrm{cm}$. El valor $2870 \mu \mathrm{S} / \mathrm{cm}$ (número de medición 1, Tabla 8, p. 6) corresponde al AMD del recipiente 1 ingresado inicialmente al sistema de tratamiento, mientras que, el valor $5861 \mu \mathrm{S} / \mathrm{cm}$ (número de medición 4, Tabla 8) corresponde al ingreso posterior de AMD del recipiente 2. A partir del ingreso del recipiente 2, con una conductividad eléctrica igual a $5861 \mu \mathrm{S} / \mathrm{cm}$ se observó un descenso hasta la medición 8 con un valor de conductividad igual a 4980 $\mu \mathrm{S} / \mathrm{cm}$, esto se debió a que el tanque inicial contenía AMD de ambos recipientes, sin embargo en las me-diciones posteriores el nivel de conductividad incre-menta hasta mantenerse a un valor aproximado del AMD contenido en el recipiente 2 .

AMD-Ca-Mg y AMD tratado: Se evidencia una tendencia de disminución de conductividad desde el AMD-Ca-Mg al AMD tratado en todas las mediciones excepto para la medición dos, tres y seis debido a condiciones operativas del sistema como rotura de manguera y/o taponamiento en las columnas por la formación de precipitados (Tabla 8 , p. 6); esta disminución se debe principalmente a la retención de metales trivalentes y divalentes que reúnen condiciones de $\mathrm{pH}$ necesarias para producir su precipitación. Sin embargo, si se analiza por separado el AMD-Ca-Mg y el AMD tratado, estos tienden a aumentar por la disolución del material reactivo de calcita $(\mathrm{Ca} 2+)$ y de óxido de magnesio $(\mathrm{Mg} 2+)$, res-pectivamente; también se incluyen los iones $\mathrm{K}+\mathrm{y} \mathrm{Na}+$ que corresponden a cationes de compensación de la zeolita [17].

Análisis de metales y aniones del sistema de tratamiento pasivo

Los resultados del análisis en laboratorio se indican en la Tabla 9 (p. 8) conjuntamente con el porcentaje de remoción calculado, en donde se debe considerar que a partir del muestreo 2, se ingresó AMD del recipiente 2 al sistema de tratamiento, además la variación de la concentración de metales y aniones del AMD inicial se debe posiblemente a las condiciones en la que se tomó la muestra en campo, la profundidad de la toma de muestra del AMD inicial, a la posible precipitación del metal en las paredes del tanque inicial, a las condiciones operativas del sistema.

\section{Aluminio (Al)}

AMD inicial y AMD tratado: Las concentraciones de Al del AMD inicial están comprendidas entre valores de un máximo de $81,50 \mathrm{mg} / \mathrm{L}$ y un mínimo de 21,50 $\mathrm{mg} / \mathrm{L}$, mientras que los valores del AMD tratado están por debajo del límite de detección del equipo. El porcentaje de remoción de aluminio se encuentra entre un valor máximo y mínimo de 99,25\% y $94,88 \%$ respectivamente; y un valor promedio de remoción del 97,81\% (Tabla 9, p. 8). Ref. [6] indican que el Al fue removido porque éste precipita como hidroxisulfato, junto con yeso debido a la eliminación de sulfato, ya que con el aumento de $\mathrm{pH}$, se integran más hidroxilos $(\mathrm{OH}-)$ y menos aniones sulfato; además el porcentaje de remoción probablemente se debió a la disolución de calcita en la columna DAS-Ca, la cual está directamente relacionada con la precipitación del Al.

Las concentraciones de Al correspondiente al AMD tratado en todos los muestreos cumplen con el criterio de calidad del TULSMA (Tabla 3, p. 5).

\section{Hierro (Fe)}

AMD inicial y AMD tratado: Las concentraciones de Fe del AMD inicial se encuentran entre un máximo de 200 mg/L y un mínimo de 14,05 mg/L, mientras que los valores de las concentraciones del AMD tratado llegan a estar situados por debajo del límite de detección del equipo. El porcentaje de remoción de hierro se encuentra entre un valor máximo y mínimo de 100\% y 99,29\% respectivamente, y un valor pro-medio de remoción del 99,79\% (Tabla 9, p. 8).

La remoción del hierro se da por varios procesos indicados por, algunos de ellos atribuidos a diferentes procesos de oxidación (SCOOFI, oxidación biótica y abiótica) [18] y la precipitación de Schwertmannita (Fe8O8(OH)5.5(SO4)1.25) [6]. Otro estudio demuestra que la eliminación del hierro sucede por la formación del mineral Goethita $(\mathrm{Fe} 3+\mathrm{O}(\mathrm{OH}))$ [5].

La remoción de este metal (al igual que el Al) se da 


\begin{tabular}{|c|c|c|c|c|c|c|c|c|c|}
\hline No. Muestreo & $\begin{array}{c}\text { Punto } \\
\text { mues- } \\
\text { treo }\end{array}$ & Al mg/L & Fe mg/L & $\begin{array}{c}\mathrm{Cu} \\
\mathrm{mg} / \mathrm{L}\end{array}$ & $\begin{array}{c}\mathrm{Pb} \\
\mathrm{mg} / \mathrm{L}\end{array}$ & Zn mg/L & Mn mg/L & $\mathrm{Cd} \mathrm{mg/L}$ & $\mathrm{SO}_{4}{ }^{2-} \mathrm{mg} / \mathrm{L}$ \\
\hline \multirow{2}{*}{1} & $\begin{array}{l}\text { AMD } \\
\text { inicial }\end{array}$ & 25,20 & 200 & 4,12 & 0,06 & 19,56 & 12,86 & 0,08 & 1936,41 \\
\hline & $\begin{array}{l}\text { AMD } \\
\text { tratado }\end{array}$ & $<0,19$ & $<0,01$ & $<0,01$ & $<0,01$ & $<0,01$ & 0,75 & $<0,01$ & 1087,79 \\
\hline \multicolumn{2}{|c|}{ Remoción (\%) } & 99,25 & 100,00 & 99,76 & 83,33 & 99,95 & 94,17 & 87,50 & 43,82 \\
\hline \multirow{2}{*}{2} & $\begin{array}{l}\text { AMD } \\
\text { inicial }\end{array}$ & 21,50 & 118,15 & 9,32 & $<0,19$ & 7,23 & 9,25 & 0,63 & 5291,63 \\
\hline & $\begin{array}{l}\text { AMD } \\
\text { tratado }\end{array}$ & $<1,1$ & $<0,1$ & $<0,08$ & $<0,19$ & 0,03 & 0,45 & $<0,03$ & 1624,46 \\
\hline \multicolumn{2}{|c|}{ Remoción (\%) } & 94,88 & 99,92 & 99,14 & - & 99,54 & 95,14 & 95,24 & 69,30 \\
\hline \multirow{2}{*}{3} & $\begin{array}{l}\text { AMD } \\
\text { inicial }\end{array}$ & 81,5 & 279,25 & 6,64 & $<0,19$ & 42,08 & 31,25 & 0,15 & 5973,52 \\
\hline & $\begin{array}{l}\text { AMD } \\
\text { tratado }\end{array}$ & $<1,1$ & $<0,1$ & $<0,08$ & $<0,19$ & $<0,02$ & 0,51 & $<0,03$ & 2918,97 \\
\hline \multicolumn{2}{|c|}{ Remoción (\%) } & 98,65 & 99,96 & 98,80 & - & 99,95 & 98,37 & 80,00 & 51,13 \\
\hline \multirow{2}{*}{4} & $\begin{array}{l}\text { AMD } \\
\text { inicial }\end{array}$ & 71 & 14,05 & 0,25 & $<0,19$ & 28,97 & 29 & 0,35 & 6907,94 \\
\hline & $\begin{array}{l}\mathrm{AMD} \\
\text { tratado }\end{array}$ & $<1,1$ & $<0,1$ & $<0,08$ & $<0,19$ & $<0,02$ & 0,9 & $<0,03$ & 4033,42 \\
\hline \multicolumn{2}{|c|}{ Remoción (\%) } & 98,45 & 99,29 & 68,00 & - & 99,93 & 96,90 & 91,43 & 41,61 \\
\hline
\end{tabular}

en la columna DAS-Ca, esto se debe al aumento de $\mathrm{pH}$ por la disolución de la calcita que favorece la precipitación de metales trivalentes.

La zeolita tiene un papel importante en la columna DAS-Mg, puesto que estudios realizados a escala de laboratorio [10], indican que el porcentaje de remoción de Fe3+ fue de 59,9\%, obteniéndose una concentración final de Fe3+ de 160,6 mg/L, después de 6 horas de contacto de $100 \mathrm{~mL}$ de solución con $3,7 \mathrm{~g}$ de zeolita. Se concluye además que la eliminación de los iones de metales pesados no sólo se debe al intercambio iónico sino también a la precipitación de hidróxidos metálicos desde la solución.

Ref. [9] demuestra que existen eficiencias de remoción de metales en solución, como el hierro, en un orden del $71 \%$ al $99 \%$, debido a la capacidad de adsorción de la zeolita por intercambio iónico entre iones del metal pesado y los cationes intercambiables en la estructura de la zeolita.

Las concentraciones de Fe correspondiente al AMD tratado cumplen con el criterio de calidad del TULSMA (Tabla 3, p. 5).

\section{Cobre (Cu)}

AMD inicial y AMD tratado: Las concentraciones de $\mathrm{Cu}$ del $\mathrm{AMD}$ inicial se encuentran entre un máximo de 9,32 mg/L y un mínimo de 0,25 mg/L, mientras que los valores de las concentraciones del AMD tratado llega a estar por debajo del límite de detección del equipo. El porcentaje de remoción de cobre se encuentra entre un valor máximo y mínimo de $99,76 \%$ y $68 \%$ respectivamente, y un valor promedio de remoción del 91,42\% (Tabla 9, p. 8).

El $\mathrm{Cu}$ se encuentra relacionado con el hierro y aluminio, dado que la remoción de Cu posiblemente ocurre por procesos de adsorción y/o coprecipitación con los precipitados de Fe y Al [19], en forma de sulfato de cobre [6]. Ref. [10] indican que el porcentaje de remoción del ion Cu2+ por zeolita natural fue de $56,8 \%$ después de 6 horas de contacto de $100 \mathrm{~mL}$ de solución con 3,7 g de zeolita. También en un estudio posterior a escala de laboratorio [9], se reportan porcentajes de remoción de cobre entre $97 \%$ y $99 \%$. Las concentraciones de Cu correspondientes a todos los muestreos del AMD tratado cumplen con el criterio de calidad del TULSMA (Tabla 3, p. 5).

\section{Plomo (Pb)}

AMD inicial y AMD tratado: Las concentraciones de $\mathrm{Pb}$ del $\mathrm{AMD}$ inicial se encuentran entre $0,19 \mathrm{mg} / \mathrm{L}$ y $0,06 \mathrm{mg} / \mathrm{L}$, mientras que los valores de las concentraciones del AMD tratado están por debajo del límite de detección del equipo de EAA. El porcentaje de remoción de plomo comprende un valor de 83,33\% en el primer muestreo del sistema de tratamiento. En 
los muestreos siguientes no fue posible realizar el cálculo del porcentaje de remoción debido a que no fue detectado por el equipo (Tabla 9, p. 8).

Ref. [19] indica que la eliminación del Pb (al igual que el $\mathrm{Cu}$ ) posiblemente ocurre por procesos de adsorción y/o coprecipitación con los precipitados de Fe y Al. Ref. [20] demostraron que este metal precipita como sulfato o fosfato de plomo a $\mathrm{pH}$ alrededor de 6,0 a 6,6; obteniéndose eficiencias de remoción altas.

Ref. [21] reportan en un estudio a escala laboratorio la eliminación casi total de $\mathrm{Pb}$ a $\mathrm{pH}$ 5,5 (PR=99,9\%) y a $\mathrm{pH} 2,2$ (PR=99,5\%), como consecuencia de la adición de zeolita. Estos resultados confirman la capacidad de la zeolita para obtener una alta eficiencia de remoción de este metal.

Las concentraciones de $\mathrm{Pb}$ en todos los muestreos cumplen con el criterio de calidad del TULSMA (Tabla 3, p. 5).

\section{Cinc (Zn)}

AMD inicial y AMD tratado: Las concentraciones de Zn del AMD inicial se encuentran entre un máximo de 42,075 mg/L y un mínimo de 7,225 mg/L, obteniendo valores finales en el AMD tratado que oscilan entre 0,03 mg/L y 0,01 mg/L. El porcentaje de remoción de cinc se encuentra entre un valor máximo y mínimo de $99,95 \%$ y $99.54 \%$ respectivamente, y un valor promedio de remoción del 99,84\% (Tabla 9 p. 8).

La remoción de Zn en la columna DAS-Mg podría deberse a la formación de minerales de retención como la Hidrocincita $\left(\mathrm{Zn}_{5}\left(\mathrm{CO}_{3}\right)_{2}(\mathrm{OH})_{6}\right)$ [22]. De igual manera el $\mathrm{Zn}$ precipita en toda la zona de la disolución de $\mathrm{MgO}$ como $\beta-\mathrm{Zn}(\mathrm{OH})_{2}$ y que cerca de la entrada de la columna DAS-Mg una pequeña cantidad de $Z n$ precipita como Hidrocincita, redisolviendo previamente $\beta-\mathrm{Zn}(\mathrm{OH})_{2}$ precipitada [8].

Ref. [21] muestran que la eliminación del cinc en condiciones de $\mathrm{pH}$ 5,5 alcanza el 93,6\% de remoción, mientras que a pH más bajo $(\mathrm{pH} 2,2)$ alcanza una remoción del 23,4\%; evidencia que permite afirmar que a mayor pH de la solución mayor porcentaje de remoción de dicho metal. Un estudio posterior reporta que el porcentaje de remoción de Zn fue de $67,8 \%$, obteniéndose una concentración final de $\mathrm{Zn}$ de 38,6 mg/L, después de 6 horas de contacto de $100 \mathrm{~mL}$ de solución con 3,7 g de zeolita; concluyendo además que la eliminación de los iones de metales pesados no sólo se debe al intercambio iónico sino también a la precipitación de hidróxidos metálicos de la solución [10]. Sin embargo un estu- dio indica que la remoción de cinc utilizando zeolita natural no fue eficiente, puesto que su porcentaje de remoción osciló entre el 34\% y 44\% [9].

Las concentraciones de Zn correspondiente al AMD tratado cumplen con el criterio de calidad del TULSMA (Tabla 3, p. 5).

\section{Manganeso (Mn)}

AMD inicial y AMD tratado: Las concentraciones de $\mathrm{Mn}$ del AMD inicial se encuentran entre un valor máximo de 31,25 mg/L y un mínimo de 9,25 mg/L mientras que los valores de las concentraciones del AMD tratado oscilan entre $0,9 \mathrm{mg} / \mathrm{L}$ y $0,45 \mathrm{mg} / \mathrm{L}$. El porcentaje de remoción de manganeso se encuentra entre un valor máximo y mínimo de 98,37\% y $94,17 \%$ respectivamente, y un valor promedio de remoción del 96.14\% (Tabla 9, p. 8).

La eliminación de este metal pudo deberse a la presencia de Hidrocincita $(\mathrm{Zn} 5(\mathrm{CO} 3) 2(\mathrm{OH}) 6)$ que favorece la formación de Loseyita ((Mn,Zn)7(CO3)2(OH)10) dado que en estos minerales se han encontrado altas concentraciones de Mn [19]. También es un estudio previo, se indica que el Mn2+ se remueve debido a la oxidación a Mn3+ que promueven las partículas de óxido de magnesio colocadas en la columna DAS-Mg [8].

Ref. [10] indican que el porcentaje de remoción de $\mathrm{Mn}^{2}+$ fue de 18,9\%, obteniéndose una concentración final de $\mathrm{Mn}^{2}+$ de $16,2 \mathrm{mg} / \mathrm{L}$, después de 6 horas de contacto de $100 \mathrm{~mL}$ de solución con $3,7 \mathrm{~g}$ de zeolita, concluyendo además que la eliminación de los iones de metales pesados no sólo se debe al intercambio iónico sino también a la precipitación de hidróxidos metálicos de la solución. Así mismo en un estudio posterior con el uso de zeolita, demuestra que hubo eficiencias de remoción de manganeso en un orden del 17\% al 33\% [9].

Las concentraciones de $\mathrm{Mn}$ correspondiente al AMD tratado cumplen con el criterio de calidad del TULSMA (Tabla 3, p. 5).

\section{Cadmio (Cd)}

AMD inicial y AMD tratado: Las concentraciones de Cd del AMD inicial se encuentran entre un máximo de 0,63 mg/L y mínimo de 0,08 mg/L, mientras que las concentraciones del AMD tratado llega a valores por debajo del límite de detección del equipo. El porcentaje de remoción de cadmio se encuentra entre un valor máximo y mínimo de 95,24\% y 80\% respectivamente, y un valor promedio de remoción del 88.54\% (Tabla 9, p. 8).

La eliminación de $\mathrm{Cd}$ en el sistema de tratamiento 
debe alcanzar un pH entre 8,5 y 10 para una total eliminación de este metal, valor que durante el experimento fue alcanzado en el punto AMD-tratado. Por otra parte, la eliminación de $\mathrm{Cd}$ se debe a la precipitación de carbonato de cadmio $\left(\mathrm{CdCO}_{3}\right)$ y de hidróxido de cadmio $\left(\mathrm{Cd}(\mathrm{OH})_{2}\right)[16]$.

La concentración del muestreo 1 correspondiente al AMD tratado se encuentra bajo el límite máximo permisible cumpliendo con la normativa ambiental nacional (Tabla 3, p. 5). Sin embargo, los muestreos posteriores se encuentran, en apariencia, sobre la normativa ambiental, esto se debe a que los valores obtenidos en estos muestreos se encuentran por debajo del límite de detección del equipo.

\section{Sulfatos $\left(\mathrm{SO}_{4}{ }^{2}-\right)$}

AMD inicial y AMD tratado: Las concentraciones de $\mathrm{SO}_{4}{ }^{2}$ - del AMD inicial se encuentran entre un valor máximo de 6907,94 mg/L y un mínimo de 1936,41 $\mathrm{mg} / \mathrm{L}$, mientras que los valores de las concentraciones del AMD tratado oscilan entre 1087,79 y 4033,42 mg/L. El porcentaje de remoción del anión sulfato se encuentra entre un valor máximo y mínimo de $69,30 \%$ y $41,61 \%$ respectivamente, y un valor promedio de remoción del 51,47\% (Tabla 9, p. 8).

La remoción de sulfatos se debe principalmente a que en la columna DAS-Mg ocurre la remoción de metales como el $\mathrm{Zn}, \mathrm{Mn}, \mathrm{Cd}$, Co y Ni que precipitan en forma de hidróxidos o hidróxido-sulfatos [5], [16]. También, en estudios previos se ha detectado la presencia de precipitados de sulfato-hidróxido de Zn-Cu (Bachererita) y Fluorita, algunos de ellos sobre fragmentos tanto en la columna DAS-Ca como en la columna DAS-Mg; además se han encontrado precipitados de sulfato de Mg [23].

Otros estudios muestran que algunos metales responsables de la retención de sulfato son el Zn que precipita en forma de sulfato de cinc [24]; Pb en forma de sulfato de plomo [20]; Cu en forma de sulfato de cobre [6]; Al en forma de hidroxisulfatos [7], [8] y Fe en forma de Schwertmannita [18]. Las concentraciones correspondientes a todos los muestreos en el AMD tratado cumplen con el criterio de calidad del TULSMA (Tabla 3, p. 5).

\section{Conclusiones}

El tratamiento de Drenaje Ácido de Mina (AMD) con el uso de zeolita natural a escala experimental, conforme a los resultados obtenidos, ha demostrado una alta remoción de metales presentes en las muestras de agua de la Quebrada "El Panteón", Parroquia Torata del Cantón Santa Rosa, Provincia de El Oro.
El pH del AMD tratado comprende valores máximo y mínimo de 8,66 y 7,59 respectivamente, obteniendo un valor promedio de 8,03 que cumple con la normativa ambiental nacional vigente correspondiente a la Tabla 9: Límites de descarga a un cuerpo de agua dulce, Reforma al Anexo 1, Libro VI del TULSMA, Acuerdo Ministerial 097.

La conductividad del AMD tratado comprende valores máximo y mínimo de $4715 \mu S / \mathrm{cm}$ y $1275 \mu \mathrm{S} / \mathrm{cm}$ respectivamente, obteniéndose un valor promedio de $3254 \mu \mathrm{S} / \mathrm{cm}$; la tendencia muestra generalmente un aumento debido posiblemente a la disolución del material reactivo de calcita y óxido de magnesio en forma de iones $\mathrm{Ca}^{2}+\mathrm{y} \mathrm{Mg}^{2}+$ respectivamente, incluido los iones $\mathrm{K}+$ y $\mathrm{Na}+$ que corresponden a cationes de compensación de la zeolita natural, además de la alta concentración de sulfatos encontrados a partir del muestreo 2, en donde se ingresó AMD del recipiente 2 al sistema de tratamiento.

La concentración de los metales pesados: hierro, aluminio, cobre, plomo, cinc, manganeso y cadmio, medidos en el AMD tratado, se encuentran bajo el límite máximo permisible establecido en la normativa ambiental nacional vigente correspondiente a la Tabla 9: Límites de descarga a un cuerpo de agua dulce, Reforma al Anexo 1, Libro VI del TULSMA, Acuerdo Ministerial 097. Sin embargo, la concentración del anión sulfato y de cadmio (muestreos 2, 3 y 4) presentan valores sobre el límite máximo permisible por dicha normativa.

\section{Recomendaciones}

Realizar investigaciones de materiales alternativos para la remoción de metales pesados y aniones del Drenaje Ácido de Mina (AMD), a escala experimental, por ejemplo el uso de roca contenida en las escombreras producto de las actividades mineras.

Incrementar la proporción de lecho de zeolita natural, disminuyendo la cantidad de adición de óxido de magnesio en la columna denominada DAS-Mg, a fin de obtener sistemas de tratamiento pasivos menos costosos.

Aplicar y evaluar el sistema de tratamiento pasivo propuesto en los diferentes pasivos ambientales mineros del Ecuador que contienen Drenaje Ácido de Mina (AMD), para la remoción de metales pesados y aniones a escala experimental.

Realizar investigaciones acerca del posible aprovechamiento de los residuos generados en las columnas de lixiviación del sistema de tratamiento. 


\section{Referencias bibliográficas}

[1] International Network for Acid Prevention. (2014). "Global Acid Rock Drainage Guide" en INAP: The International Network for Acid Prevention. [En línea]. Disponible en: http:// www.gardguide.com/images/5/5f/TheGlobalAcidRockDrainageGuide.pdf [Revisado el día 20 de febrero de 2017]

[2] Delgado Rodríguez, J., (2012) Estudio de la contaminación por metales pesados en la Cuenca Baja del río Guadiana. Tesis doctoral. Huelva. Universidad de Huelva, Departamento de Geología, Facultad de Ciencias Experimentales.

[3] Nyquist, J. y Greger, M., (2009). "A field study of constructed wetlands for preventing and treating acid mine drainage" en Ecological Engineering, No. 35, pp. 630-642.

[4] Lottermoser, B.G., (2010) Mine Wastes: Characterización, Treatment and Environmental Impacts. 3era edición. Springer.

[5] Caraballo, M., Rotting, T.S., Macías, F., Nieto, J.M. y Ayora, C., (2009). "Field multi-step limestone and MgO passive system to treat acid mine drainage with high metal concentrations" en Applied Geochemistry. No. 24, pp. 2301-2311.

[6] Rotting, T.S., Thomas, R.C., Ayora, C. y Carrera, J., (2008a). "Passive Treatment of Acid Mine Drainage with High Metal Concentrations Using Dispersed Alkaline Substrate" en Journal of Environmental Quality, No. 37, pp. 1741-1751.

[7] Cortina, J.L., Lagreca I. y Pablo, J., (2003). "Passive In Situ Remediation of Metal-Polluted Water with Caustic Magnesia: Evidence from Column Experiments" en Environmental Science and Technology. No. 37, pp. 1971-197.

[8] Rotting, T.S., J.A., Ayora, C. y Carrera, J., (2008c). "Improved Passive Treatment of High Zn and Mn Concentrations Using Caustic Magnesia (MgO): Particle Size Effects" en Environmental Science and Technology, No. 42, pp. 93709377.

[9] Motsi, T., (2010). Remediation of Acid Mine Drainage Using Natural Zeolite. Tesis doctoral. Escuela de Ingeniería Química, Universidad de Birmingham, Reino Unido.

[10] Motsi, T., Rowson, N.A. y Simmons, M.J.H., (2009). "Adsorption of heavy metals from acid mine drainage by natural zeolite" en International Journal of Mineral Processing, No. 92, pp. 42-48.

[11] Wingenfelder, U., Hansen, C., Furrer, G. y Schulin, R., (2005). "Removal of Heavy Metals form Mine Waters by Natural Zeolites" en Environmental Science and Technology, No. 39, pp. 4606-4613.

[12] Ministerio del Ambiente del Ecuador, (Noviembre de 2015). Acuerdo Ministerial No. 097 Sustitúyase el Libro VI del Texto Unificado de Legislación Secundaria. Quito, Ecuador: Registro Oficial No.387.

[13] Morin, K. y Hutt, N. (2001). "Relocation of net-acid-genera- ting waste to improve post-minig wáter chemistry" en Waste Management, No. 21, pp. 185-190.

[14] Aduvire, O. (2006). "Drenaje ácido de mina generación y tratamiento". Madrid, disponible en: http://info.igme.es/SIDIMAGENES/113000/258/113258_0000001.PDF [Revisado el día 20 de febrero de 2017]

[15] Leinonen, H. y Lehto, J., (2001). "Purification of metal finishing waste waters with zeolites and activated carbons" en Waste Management and Research, No. 19, pp. 45-57.

[16] Rotting, T.S., Cama, J., Ayora, C., Cortina, J.L. y De Pablo, J., (2006). "Use of Caustic Magnesia To Remove Cadmium, Nickel, and Cobalt from Water in Passive Treatment Systems: Column Experiments" en Environmental Science and Technology, No. 40, pp. 6438-6443.

[17] Bustillos Yaguana, A. y Suin Arévalo, M., (2014) Diseño y construcción de un reactor químico para la obtención de zeolitas sintéticas. Tesis de ingeniería. Cuenca, Universidad de Cuenca, Facultad de Ciencias Químicas, Escuela de Ingeniería Química.

[18] Macías, F., Caraballo, M.A., Nieto, J.M., Rotting T.S. y Ayora, C., (2012). "Natural pretreatment and passive remediation of highly polluted acid mine drainage" en Journal of Environmental Management, No. 104, pp. 93-100.

[19] Macías, F., (2013) Estudio mineralógico y geoquímico de la optimización de un sistema de tratamiento pasivo de drenaje ácido de mina con alta carga metálica. Tesis doctoral. Huelva. Universidad de Huelva, Facultad de Ciencias Experimentales, Departamento de Geología.

[20] Oliva, J., De Pablo, J., Cortina, J.L., Cama, J. y Ayora, C., (2010). "The use of Apatite IITM to remove divalent metal ions zinc (II), lead (II), manganese (II) and iron (II) from water in passive treatment systems: columns experiments" en Journal of Hazardous Materials, No. 184. pp. 364-374.

[21] Wingenfelder, U., Hansen, C., Furrer, G. y Schulin, R., (2005). "Removal of Heavy Metals form Mine Waters by Natural Zeolites" en Environmental Science and Technology, No. 39, pp. 4606-4613.

[22] Pérez López, R., Macías, F., Caraballo, M.A., Nieto, J.M., Román Ross, G., Tucoulou, R. y Ayora, C., (2011). "Mineralogy and Geochemistry of Zn-Rich Mine-Drainage Precipitates From an MgO Passive Treatment System by Synchrotron-Based X-ray Analysis" en Environmental Science and Technology, No. 45, pp. 7826-7833.

[23] Piris Casanova, G., (2015). Tratamiento de Aguas Ácidas de Mina. Trabajo fin de carrera. Barcelona. Departamento de Ingeniería del Terreno, Cartográfica y Geofísica.

[24] Micera, G., Gessa, C., Melis P., Premoli, A., Dallocchio, R. y Deeana, S., (1986). "Zinc (II) Adsorption on aluminum hydroxide" en Colloide and Surface, No. 17, pp. 389-394 